\title{
Pigment effect on the long term elasticity of elastomeric ligatures
}

\author{
Érika de Oliveira Dias de Macêdo', Fabrício Mezzomo Collares², Vicente Castelo Branco Leitune, \\ Susana Maria Werner Samuel ${ }^{4}$, Carmen Beatriz Borges Fortes ${ }^{5}$
}

Objective: To evaluate the response of elastomeric ligatures in several colors for a $4 \mathrm{~mm}$ traction over time.

Methods: Morelli ${ }^{\circledR}$ elastomeric ligatures, were submitted to traction forces using two rods of circular cross section, until a $4 \mathrm{~mm}$ distance was reached, matching the approximate diameter of an upper central incisor bracket of the same manufacturer. The ligatures were kept in artificial saliva immersion at $37^{\circ} \mathrm{C}$. Forces levels were measured immediately ( $0 \mathrm{~h}), 2,4,6,8,10,12,24,48,72,96$ hours, 1, 2, 3, 4 weeks and results were submitted to two-way repeated-measures ANOVA statistical analysis.

Results: The gray samples showed the higher initial values of tensile strength. The lowest values were presented by purple, light pink, green, black and red groups. The greater tensile strength instability was presented by red, black, silver, green and gray groups. The greater tensile strength stability was presented by deep pink, dark blue, blue, purple and light pink groups.

Conclusion: Elastomeric ligatures do not present stable behavior when suffering traction forces over time and different colors display different behaviors. Deep pink, dark blue, blue, purple and light pink groups, displayed the most stable forces, suggesting that they should be used during the treatment to obtain constant forces.

Keywords: Ligatures. Elastomers. Color. Elasticity.

${ }^{1} \mathrm{PhD}$ student in General Dentistry with emphasis in Dental Materials, UFRGS.

${ }^{2}$ Associate Professor of Dental Materials, UFRGS.

${ }^{3} \mathrm{PhD}$ student in General Dentistry with emphasis in Dental Materials, UFRGS

${ }^{4}$ Head Professor of Dental Materials, UFRGS.

${ }^{5}$ Associate Professor of Dental Materials, UFRGS.
How to cite this article: Macêdo EOD, Collares FM, Leitune VCB, Samuel SMW, Fortes CBB. Pigment effect on the long term elasticity of elastomeric ligatures. Dental Press J Orthod. 2012 May-June;17(3):27.el-6.

Submitted: April 26, 2009 - Revised and accepted: April 12, 2010

» The authors report no commercial, proprietary, or financial interest in the products or companies described in this article.

Contact address: Érika de Oliveira Dias de Macêdo Rua Ramiro Barcelos, 2492 - Santana

Zip code: 09.0035-003 - Porto Alegre/RS, Brazil

E-mail: erikaodias@gmail.com 


\section{INTRODUCTION}

Elastomeric ligatures are used during dental alignment and leveling stages, allowing the arch wires of small diameters to be connected to the teeth generating horizontal, both bucco-lingual and mesial-distal, and vertical movement. The elastomeric ligatures are also used for rotation correction and retractions. Furthermore, the possibility of choosing the ligatures color facilitates the adhesion of young people to treatment, as there is a vast option of colors available in the market nowadays.

Elastomeric ligatures are polyurethane polymers produced by a step-reaction polymerization process with cross-linking connection that allow the elastic recovery to its initial spiral pattern. However, despite having elastic properties, ligatures are not considered perfect elastics, as they suffer structural deformation of the polymer chain, what leads to permanent deformation. This phenomenon is called force relaxation and it consists in the decay of the necessary force to distend the elastomeric ligatures over time. . $^{1,7,8,15}$

Pigments that are incorporated to ligatures' elastomeric matrix to give the material different colors seem to affect its mechanical properties, however few studies have addressed this issue., ${ }^{1,2}$ The aim of this study was to evaluate the behavior of elastomeric ligatures of different colors when they are submitted to traction after different storage periods.

\section{MATERIAL AND METHODS}

Elastomeric ligatures (Morelli ${ }^{\circledR}$ ), in ten different colors were used in this study: light green, red, light pink, purple, deep pink, blue, dark blue, black, gray and silver. Each color represents a group of 10 units of elastomeric ligatures. As the main function of the ligatures is to connect the archwire to the bracket, keeping the wire under constant pressure to promote tooth movement, the diameter of a Morelli ${ }^{\circledR}$ central incisor bracket was used as standard, which is equivalent to the average distance of the ligature tier used for this purpose, $4 \mathrm{~mm}$.

Ligature traction was performed in a universal testing machine EMIC DL 2000 (EMIC São José dos Pinhais, PR - Brazil), with the aid of a device comprised of two stainless steel "L" shaped rods with circular cross section of $0.7 \mathrm{~mm}$ (Fig 1). The angled portions of the stems were positioned together and introduced into the ligatures that were then submitted to traction at a speed rate of $1 \mathrm{~mm} / \mathrm{s}$ until the internal diameter of the ligature ( $1.5 \mathrm{~mm}$ at rest) would reach $4 \mathrm{~mm}$. The force, in Newtons, necessary to elongate the ligature of each group was immediately registered $(0 \mathrm{~h})$ and then registered again after storage period of $2,4,6,8,10,12,24$, $48,72,96$ hours and 1, 2, 3, 4 weeks. Samples were kept in artificial saliva at $37^{\circ} \mathrm{C}$.

Statistical Analysis was performed by two-way (color and time) repeated-measures ANOVA. The statistical significance level was of $95 \%$.

\section{RESULTS}

The elastomeric ligatures' behavior during the traction test is shown in Figure 2 and Table 1. The gray pigmented elastomeric ligatures presented higher initial forces when submitted to traction in comparison to the purple, light pink, green, black and red groups. However there was no significant difference among the force values for the deep pink, dark blue, blue and silver groups.

At hour 2 the gray group still presented the highest force values showing statistically significant difference when compared to the deep pink, light pink

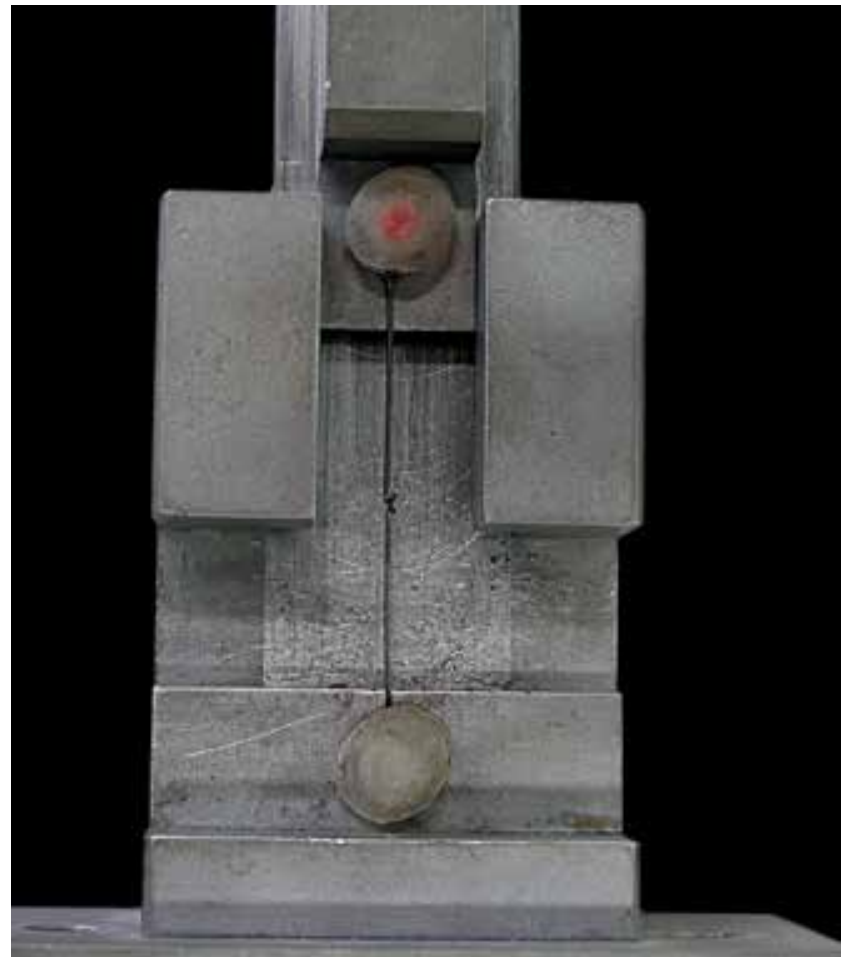

Figure 1- Device in stainless steel used to attach the ligatures during tests. 
and black groups. At week 1, the green, black, gray and silver groups presented statistically significant force decay when submitted to the traction test in comparison to the other groups. At that moment, the gray group was only different from the light pink and red groups.

There was no significant force reduction over time for the purple, light pink, dark blue, blue and deep pink groups, displaying a more constant behavior. The green group was the only one that presented significant force decay when submitted to traction at week 4 in relation to its initial values. The red, green and black groups displayed the greatest force oscillations during the testing period, varying between force increases and decreases.

\section{DISCUSSION}

Polyurethane elastics have been widely used in orthodontics and they have replaced the latex ones in cases of hyper-sensible patients. However, they are not considered ideal elastics and behave in a non-uniform way. Part of their force is lost over the time, what can interfere in tooth movement within the 28 days they are generally in intraoral use. Moreover, different behaviors have been observed among the various color the elastomeric ligatures are made in, and some studies have suggested that the manufacture process and the kinds of pigments used may influence the material's structure and, consequently, its capacity of traction resistance over time. , $^{1,2,11,12}$

Table 1- Mean and standard deviation for the force values $(\mathrm{N})$ obtained by traction for the different colors and intervals.

\begin{tabular}{|c|c|c|c|c|c|c|c|c|c|c|}
\hline $\mathbf{s}$ & $y$ & K & e & le & $r$ & & & & k & d \\
\hline & $\begin{array}{l}(40 . \\
B, a\end{array}$ & $\mathrm{~B}, \mathrm{a}$ & $\mathrm{B}, \mathrm{C}, \mathrm{a}$ & $\begin{array}{l}6(40.17) \\
B, C, a\end{array}$ & $\begin{array}{l}14(40.17) \\
B, C, a\end{array}$ & $\mathrm{C}, \mathrm{a}$ & $\mathrm{C}, \mathrm{a}$ & $\mathrm{C}, \mathrm{a}$ & $\begin{array}{l}5(40.19) \\
C, a\end{array}$ & $\begin{array}{l}4(40 . \\
C, a\end{array}$ \\
\hline 2 & $B, b, c$ & $C, D$, & $B, C$ & $\begin{array}{l}05(40.16) \\
B, C, D, b\end{array}$ & $\begin{array}{l}02(40.16) \\
B, C, D, b\end{array}$ & $\mathrm{~B}, \mathrm{C}$ & $C, D, b$ & $\mathrm{~B}, \mathrm{C}$ & $C, D$, & $B, D, b, c$ \\
\hline $4 \mathrm{~h}$ & $B, C, b$ & $\mathrm{C}, \mathrm{b}$ & $B$, & $\mathrm{C}, \mathrm{b}$ & $\begin{array}{l}2(40.13) \\
B, C, b\end{array}$ & $c, b$ & C & $\mathrm{B}$, & $\begin{array}{c}95(40.17) \\
C, b, c\end{array}$ & $c, b, c$ \\
\hline 6 & $B, b, c$ & $\mathrm{~B}, \mathrm{C}, \mathrm{b}$ & $\mathrm{B}, \mathrm{C}$ & $\begin{array}{c}94(40.14) \\
B, C, b\end{array}$ & $\begin{array}{c}84(40.14) \\
B, C, b, C\end{array}$ & $B, C, b$ & $\mathrm{~B}, \mathrm{C}$ & $B, C, b$ & $\mathrm{~B}, \mathrm{C}$ & $c, b, c$ \\
\hline 8 & $B, b, c$ & $B$, & B & $B, C, b$ & $B, C, b$, & $\mathrm{B}, \mathrm{C}$ & $\mathrm{B}, \mathrm{C}$ & $B, C$, & $B, C$ & $c, b$ \\
\hline 10 & $B, b, c$ & B, & $\mathrm{B}$ & $\begin{array}{c}92(40.12) \\
B, C, b\end{array}$ & $B, C$ & B, & B & $B$, & $\mathrm{B}$ & $c, b$ \\
\hline 12 & $B, b, c$ & $B$, & B & $\begin{array}{c}88(40.15) \\
B, C, b\end{array}$ & $\mathrm{~B}, \mathrm{C}$ & $B$, & B & $\mathrm{B}$, & $B$, & $\begin{array}{l}8(4 \\
C, b,\end{array}$ \\
\hline 24 & $B, b, c$ & $\mathrm{~B}, \mathrm{C}$ & $\mathrm{B}$ & $\begin{array}{c}94(40.13) \\
B, C, b\end{array}$ & $\begin{array}{c}85(40.15) \\
B, C, b, C\end{array}$ & $B, C$ & $\mathrm{~B}, \mathrm{C}$ & $B, C$ & $B, a$ & $c, b, c$ \\
\hline 48 & $\begin{array}{c}12(40.1 \\
B, b, c\end{array}$ & B & B & $\begin{array}{c}89(40.25) \\
B, C, b\end{array}$ & $C, b$ & $B$, & C & $B$, & $B, C$ & $c, b$ \\
\hline $72 \mathrm{~h}$ & $\begin{array}{c}.05(40.1 \\
B, b, c\end{array}$ & $B, b$ & $B$ & $\begin{array}{c}87(40.14) \\
B, C, b\end{array}$ & $B, C$ & $B$ & .21) & $B, C$ & $\mathrm{~B}, \mathrm{C}$ & $\begin{array}{c}.61(40.18) \\
C, c\end{array}$ \\
\hline $96 \mathrm{~h}$ & $\begin{array}{c}17(40.18) \\
B, b, c\end{array}$ & $B, C, b$ & $\begin{array}{r}96( \\
B, C\end{array}$ & $\begin{array}{c}96(40.14) \\
B, C, b\end{array}$ & $\begin{array}{c}.85(40.14) \\
C, b, c\end{array}$ & $\begin{array}{c}89(40.23) \\
B, C, b\end{array}$ & $\begin{array}{c}2.88(40.20) \\
C, b\end{array}$ & $\begin{array}{r}2.94(4 \\
B, C, L\end{array}$ & $B, C$, & $\begin{array}{c}.94(40.10) \\
B, C, b\end{array}$ \\
\hline 1st & $\begin{array}{c}.97(40.19) \\
B, C\end{array}$ & $\begin{array}{c}77(40.15) \\
B, C, b\end{array}$ & $B, C, b$ & $\begin{array}{c}77(40.14) \\
B, C, b\end{array}$ & $\begin{array}{c}67(40.14) \\
C, c\end{array}$ & $\begin{array}{c}73(40.24) \\
B, C, b\end{array}$ & $\begin{array}{c}.67(40.19) \\
C, b\end{array}$ & $\begin{array}{c}83(40.15) \\
B, C, C\end{array}$ & $\begin{array}{c}.75(40.14) \\
B, C, C\end{array}$ & $\begin{array}{c}2.59(40.18) \\
\text { C,C }\end{array}$ \\
\hline $2 \mathrm{nd}$ & $\begin{array}{c}.21(40.21) \\
B, b, c\end{array}$ & $B, C, b$ & $B, C, b$ & $\begin{array}{c}95(40.14) \\
C, b\end{array}$ & $\begin{array}{c}87(40.13) \\
C, b, c\end{array}$ & $\begin{array}{c}.90(40.26) \\
C, b\end{array}$ & $\begin{array}{c}2.86(40.20) \\
C, b\end{array}$ & $\begin{array}{c}.91(40.15) \\
c, b, c\end{array}$ & $\begin{array}{c}.90(40.13) \\
c, b, c\end{array}$ & $\begin{array}{c}77(40.22 \\
c, b, c\end{array}$ \\
\hline 3rd we & $\begin{array}{c}3.19(40.23) \\
B, b, c\end{array}$ & $\begin{array}{l}99(40.13) \\
B, C, b\end{array}$ & $B, C, b$ & $\begin{array}{c}98(40.14) \\
B, C, b\end{array}$ & $\begin{array}{c}2.89(40.14) \\
c, b, c\end{array}$ & $\begin{array}{c}2.91(40.25) \\
B, C, b\end{array}$ & $\begin{array}{c}2.90(40.22) \\
C, b\end{array}$ & $\begin{array}{c}.94(40.16) \\
B, C, b, C\end{array}$ & $\begin{array}{c}.97(40.15) \\
B, C, b, C\end{array}$ & $\begin{array}{c}2.73(40.21) \\
c, b, c\end{array}$ \\
\hline fln we & $\begin{array}{c}08(40.22) \\
B, b, c\end{array}$ & $\begin{array}{c}90(40.1 \\
B, C, b\end{array}$ & $\begin{array}{c}85(40.1 \\
B, C, b\end{array}$ & $\begin{array}{c}33(40.13) \\
B, C, b\end{array}$ & $\begin{array}{l}7(40.14) \\
C, b, c\end{array}$ & $\begin{array}{c}32(40.25) \\
B, C, b\end{array}$ & $\begin{array}{c}2.82(40.22) \\
B, C, b\end{array}$ & $\begin{array}{c}38(40.13) \\
B, C, C\end{array}$ & $\begin{array}{c}2.82(40.14) \\
B, C, C\end{array}$ & $\begin{array}{c}2.64(40.18) \\
C, c\end{array}$ \\
\hline
\end{tabular}

Different capital letters show statistical difference in the columns. Different lowercase letters show statistical differences in the lines. 


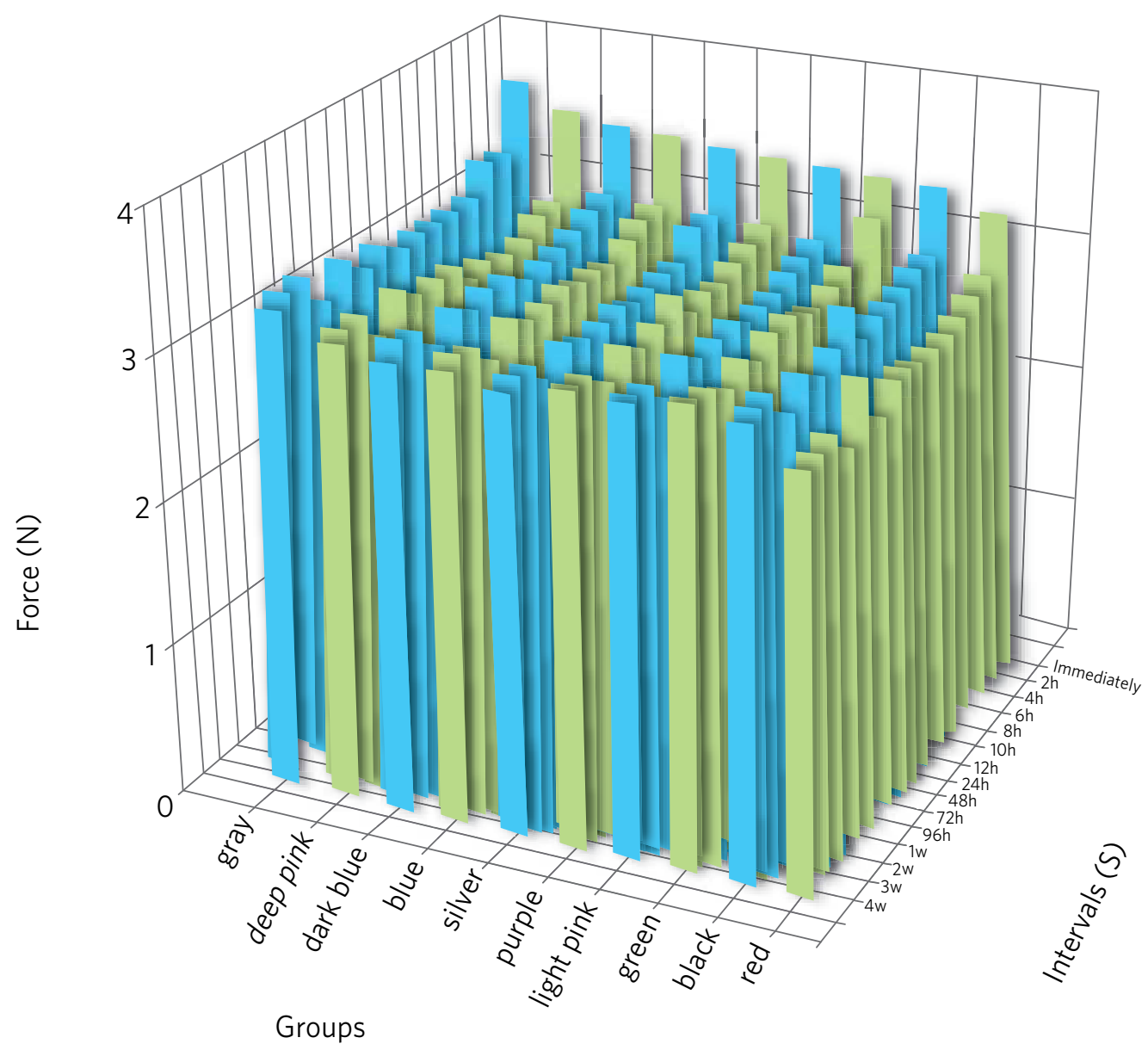

Figure 2 - Force values $(\mathrm{N})$ under $4 \mathrm{~mm}$ traction for the different groups of color, in the assessed periods.

According to the law of action-reaction the values obtained also indicate the remnant internal tension of the elastic that corresponds to its potential capacity of exerting force over the archwire to achieve the desired tooth movement.

The studies that evaluated the behavior of the elastomeric ligatures over time showed that the highest loss of traction resistance occurs within the first 24 hours ${ }^{1,3,14,15}$ and that an average of $30 \%$ of that loss occurs within the first hour, ${ }^{4,5,11,13}$ which corroborates the findings in this study. These differences may be due to: different manufacture techniques involving the cutting or the injection-moulding of the raw material; the additives incorporated to the final product; the morphological differences (ellipsoid or circular modulus) and, in the case of elastic chains, to their dimensional characteristics (the presence or absence of inter-modular connections).
There is great variation of traction resistance between elastomeric ligatures kept in wet medium and those kept in dry medium. For this reason the ligatures used in this experiment were kept in artificial saliva. ${ }^{6}$ The in vitro aging process of the polyurethane involves, initially, the degradation of the ether group, followed by the loss of the free urethane not linked to the ester group and the loss of the hydrogen linked to the urethane of the ester group and urea group. Therefore, the water hydrolyzes the ester or the ether that are bonded to the main chain, which promotes structural degradation of the polymer's chain and accelerates the aging and weakening process of the material. This process reduces the necessary force to keep the ligatures distended and, consequently, diminishes its internal tension under traction.,

Differently from the wet medium, where the aging process occurs by the hydrogen removal from beta-methylene group, leading to the loss of alcohol 
and alkenes, in the dry medium the removal of the hydrogen is carried out from the alpha-methylene group, which means a slower degradation process. ${ }^{7,8}$

Taloumis et al ${ }^{14}$ showed the influence of the storage medium on the configuration of the ligatures, proving that immersion of the elastics in wet medium itself is capable of changing their dimensions, corroborating Wong's ${ }^{15}$ findings that showed that these ligatures absorb saliva, which changes their structural configuration and their behavior when submitted to traction.

Regarding the behavior of the tension over time, some studies have reported that higher initial forces determine higher decay rates over time. ${ }^{10,13,14}$ This phenomenon was observed in this study in the gray group that obtained higher initial forces and lower stability in the maintenance of these forces when compared to the groups with intermediate initial values.

The results obtained by De Genova et al, ${ }^{5}$ showing that higher initial values had lesser force decay under traction, agree with the results found in this study for the deep pink, dark blue and blue groups, as these colors presented high initial force, with no statistical difference to the gray group, and good stability over time. The groups with lower initial values presented a more instable behavior, which is also in agreement with the results obtained by De Genova et $\mathrm{al}^{5}$ that described a higher force decay for the ligatures with lower initial force after 21 days.

The force increase from the second week on is justified by the findings of Wong, ${ }^{15}$ who revealed a probable loss of the elastic properties of the material related to time and intensity of traction, which would lead to a plastic deformation that makes the elastomeric ligatures stiffer to strain. The subsequent reduction of the traction force resistance values found in this study may be related, according to Taloums et $\mathrm{al},{ }^{14}$ to the permanent dimensional changes, to the enlargement of the internal diameter of the ligature and to the width of the wall of the external diameter that lead to force decay over time.

The variations found in this study regarding the different colors suggest that the different kinds of pigment used in the elastomeric ligatures may change the ligatures' behavior, corroborating the findings of Baty et $\mathrm{al}^{2}$ that showed differences between some brands of ligatures and explained it as being the result not only of the pigment employed, but also of the manufacturing technique employed. The results found by Lu et $\mathrm{al}^{11}$ also showed differences associated to pigment addition, finding statistically superior results for transparent elastomeric ligatures when compared to gray ones. The differences in the behavior of different colors ligatures may influence on the color choice, as the colors with better stability over time are more recommended due to their less jeopardizing biological effects.

The variation in the traction force values found in each group shows the need of an individual control, since these differences promote various effects depending on the clinical situation, the anatomical conditions and bone support of each patient.

Force decay over time may have influence particularly in cases demanding continuous full engagement of the arch wire with the bracket to achieve the desired movement, such as tooth rotation, over $3 \mathrm{~mm}$ of dental crowding, as demonstrated by Franchi and Baccetti, ${ }^{9}$ and during alignment and leveling, in which the force decay under traction generates intermittent and decreasing stimulus that may influence tooth movement negatively, particularly when using thermo-activated arch wires that act for longer periods. Considering that after 28 days the ligatures are unable to establish an ideal engagement between the arch wire and the slot, there is distortion of the arch wire`s long term effects.

\section{CONCLUSION}

Elastomeric ligatures do not present stable behavior when submitted to traction over time. The ligature's color is a determinant variable to its behavior, having groups of different colors presented different behavior. The choice of this material must be based on the desired effects in order to obtain effective outcomes over treatment and allow predictability of its effects on patients.

Within the limitations of this in vitro study, the green, black and red groups were the ones that presented higher force oscillation when submitted to traction. Among all groups, the green one presented a statistically significant reduction between the initial and final measurements of the experiment. The deep pink, dark blue, blue, purple and light pink groups did not present statistically significant differences between the initial and final measurements, what might suggest to the orthodontist that these colors would present more constant forces during treatment. 


\section{REFERENCES}

1. Baty DL, Storie DJ, von Fraunhofer JA. Synthetic elastomeric chains: a literature review. Am J Orthod Dentofacial Orthop. 1994 Jun;105(6):536-42.

2. Baty $\mathrm{DL}$, Volz JE, von Fraunhofer JA. Force delivery properties of colored elastomeric modules. Am J Orthod Dentofacial Orthop. 1994 Jul;106(1):40-6.

3. Bertoncini C, Cioni E, Grampi B, Gandini P. In vitro properties changes of latex and non-latex orthodontic elastics. Prog Orthod. 2006;7(1):76-84.

4. Bousquet JA Jr, Tuesta O, Flores-Mir C. In vivo comparison of force decay between injection molded and die-cut stamped elastomers. Am J Orthod Dentofacial Orthop. 2006 Mar;129(3):384-9.

5. De Genova DC, McInnes-Ledoux P, Weinberg R, Shaye R. Force degradation of orthodontic elastomeric chains: a product comparison study. Am J Orthod. 1985 May;87(5):377-84.

6. Dowling PA, Jones WB, Lagerstrom L, Sandham JA. An investigation into behavioural characteristics of orthodontic elastomeric modules. Br J Orthod. 1998 Aug;25(3):197-202.

7. Eliades T, Eliades G, Watts DC. Dental Materials in vivo ageing and related phenomena, Surrey (GB): Quintessence; 2003.

8. Eliades T, Eliades G, Watts DC. Structural conformation of in vitro and in vivo aged orthodontic elastomeric modules. Eur J Orthod. 1999 Dec;21(6):649-58.
9. Franchi L, Baccetti T. Forces released during alignment with a preadjusted appliance with different types of elastomeric ligatures. Am J Orthod Dentofacial Orthop. 2006 May;129(5):687-90.

10. Lam TV, Freer TJ, Brockhurst PJ, Podlich HM. Strength decay of orthodontic elastomeric ligatures. J Orthod. 2002 Mar;29(1):37-43.

11. Lu TC, Wang WN, Tarng TH, Chen JW. Force decay of elastomeric chain: A serial study. Part II Am J Orthod Dentofacial Orthop. 1993 Oct;104(4):373-7.

12. Renick MR, Brantley WA, Beck FM, Vig KW, Webb CS. Studies of orthodontic elastomeric modules. Part 1: Glass transition temperature for representative pigmented products in the as received condition an after orthodontic use. Am J Orthod Dentofacial Orthop. 2004 Sep;126(3):337-43.

13. Stevenson JS, Kusy RP. Force application and decay characteristics of untreated and treated polyurethane elastomeric chains. Angle Orthod. 1994;64(6):455-64; discussion 465-7.

14. Taloumis LJ, Smith TM, Hondrum SO, Lorton L. Force decay and deformation of orthodontic elastomeric ligatures. Am J Orthod Dentofacial Orthop. 1997 Jan;111(1):1-11.

15. Wong AK. Orthodontic elastic materials. Angle Orthod. 1976 Apr;46(2):196-205. 